\title{
DESIGN AND SYNTHESIS OF SOME P-SUBSTITUTED STYRYLISOXAZOLE CARBOXYLIC ACID DERIVATIVES AS ANTI-INFLAMMATORY AGENTS
}

Farghaly A. Omar ${ }^{1}$, Atef A. Abdel-Hafez ${ }^{1 *}$ and Montaser S. Ahmed ${ }^{2}$

${ }^{1}$ Department of Pharmaceutical Medicinal Chemistry, Faculty of Pharmacy, Assiut University, Assiut 71526, Egypt

${ }^{2}$ Department of Pharmaceutical Chemistry, Faculty of Pharmacy, Alazhar University, Assiut, Egypt

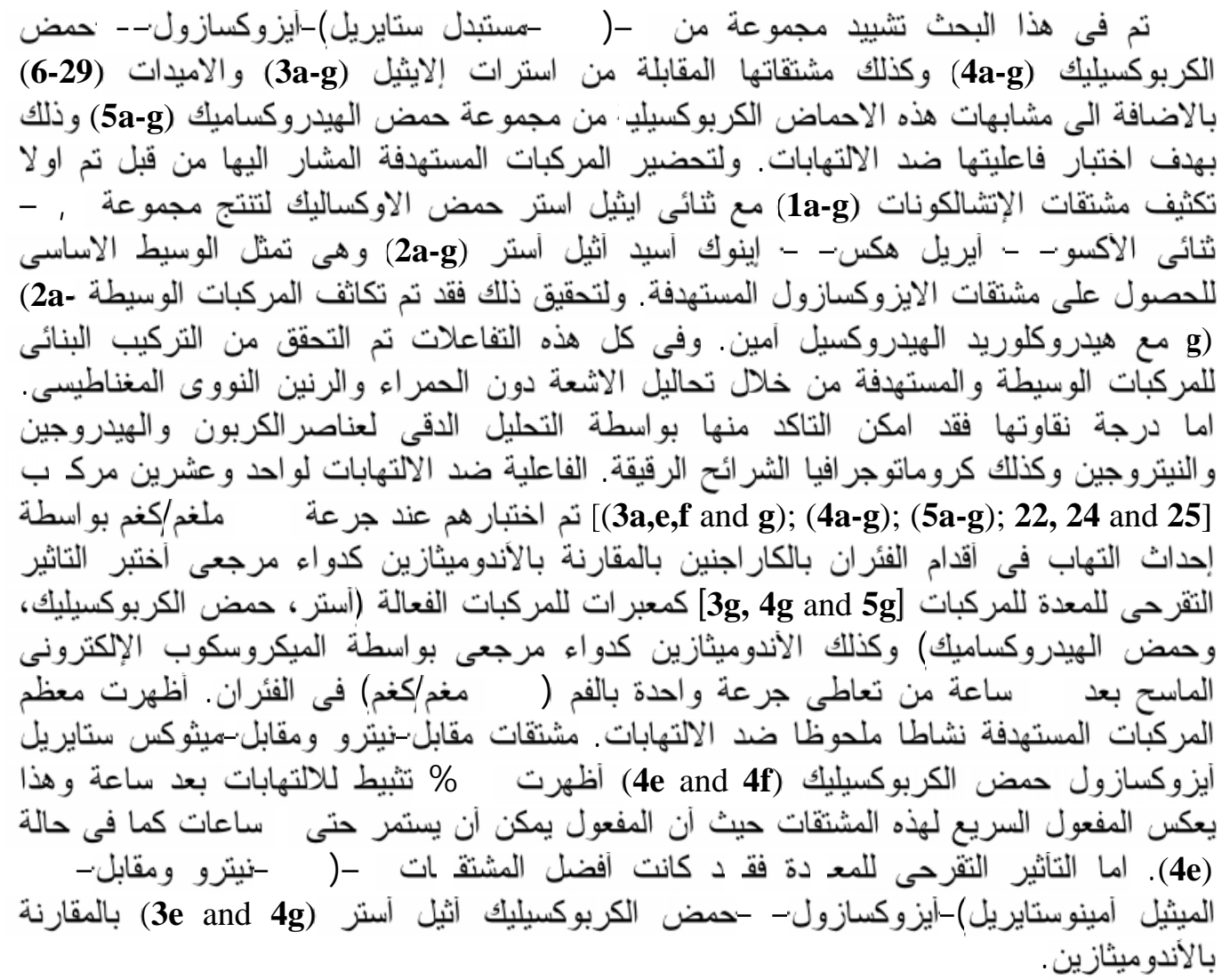

A series of 5-(p-substitutedstyryl)-isoxazole-3-carboxylic acids (4a-g), carboxylic acid ethyl esters (3a-g) and their corresponding amides (6-29) as well as their hydroxamic acid analogues (5a-g) were synthesized and evaluated for their anti-inflammatory activity. The 1,3dicarbonyl systems 2,4-dioxo-6-arylhex-5-enoic acid ethyl esters (2a-g) were the key intermediate for synthesis of the target isoxazole derivatives. The synthesis of these intermediate was achieved through the reaction of chalcones (1a-g), prepared via aldol condensation of the respective substituted benzaldehyde with acetone in alkaline medium, with diethyloxalate. The purity of the synthesized derivatives was determined by thin layer chromatography (TLC) in addition to the microanalyses and their structures were confirmed by different spectroscopic means. The anti-inflammatory activity of twenty-one compounds [(3a,e,f and $\mathbf{g}) ;(\mathbf{4 a - g}) ;(\mathbf{5 a}-\mathbf{g})$; 22; 24 and 25] were assessed, at a dose of $100 \mathrm{mg} / \mathrm{kg}$, by carrageenan-induced paw edema in rats in comparison to indomethacin as a reference drug. The ulcerogenicity of the compounds $3 g, 4 g$ and $\mathbf{5 g}$ as representatives of the anti-inflammatory active compounds (ester, carboxylic acid and hydroxamic acid) and indomethacin as reference drug was examined under scan

Received in 13/4/2004 \& Accepted in 20/5/2004

*Corresponding author, Tel: +20-88-303006, Fax: +20-88-33-2776, E. mail: atef@acc.aun.edu.eg 
electron microscope, after 24 hours of administration of single dose (100 $\mathrm{mg} / \mathrm{kg})$ in rats. Significant anti-inflammatory activity was displayed by most of the target derivatives. $p$-Nitro and p-methoxystyryl isoxazole carboxylic acid derivatives ( $4 \boldsymbol{e}$ and $\mathbf{4 f}$ ) revealed $75 \%$ inhibition of inflammation after $1 \mathrm{hr}$ reflected the rapid onset of action of these derivatives, which may sustained up to $5 \mathrm{hr}$ as in case of $4 \boldsymbol{e}$. The anti-inflammatory activity of 5-(p-nitro and pdimethylaminostyryl)-isoxazole-3-carboxylic acid ethyl esters (3e and $\mathbf{3 g})$ being favorably comparable with indomethacin in terms of potency and ulcerogenic liability. The rational behind the synthesis of these compounds and the structure activity relationship are discussed.

\section{INTRODUCTION}

Non-steroidal anti-inflammatory drugs (NSAIDs) are among the most widely used therapeutics, primarily for the treatment of pain and inflammation especially arthritis. ${ }^{1}$ The Inhibition of cyclooxygenase (COX) is a hallomark feature of virtually all marketed NSAIDs currently in wide use for the treatment of rheumatoid arthritis and osteoarthritis. ${ }^{2}$ However, NSAIDs possess certain types of mechanism-based side effects including GIT ulceration and nephrotoxicity. ${ }^{3}$ Hence, the discovery of novel dual inhibitors of COX and 5-LO appears to be a fruitful approach towards the identification of safer second-generation NSAIDs. For a long time the presence of an acidic moiety was thought to be a pre-requisite for the classical NSAIDs. Today we know that this structural feature accounts for the formation of a salt bridge with the ARG 120 at the bottom of cyclooxygenase enzyme thus generating the COX-1 inhibiting activity. ${ }^{1}$ Depending on their chemical structure, NSAIDs inhibit both COX-1 and COX-2 to different extents. This accounts for their antiinflammatory and analgesic activities and their unwanted GI side effects. ${ }^{1}$ On the other hand, hydroxamic acids were well known to form strong complexes with a variety of transition metals. This property has been exploited in the use of hydroxamates as inhibitors of several metalloenzymes. ${ }^{4}$ Since it is generally believed that 5-lipooxygenase contains a catalytically important iron atom, ${ }^{5}$ this enzyme is a logical candidate for inhibition by hydroxamic acid containing molecules. Styrylisoxazole derivatives (I) were synthesized and studied for the anti-inflammatory activity. These compounds possess anti-inflammatory activity, by dual mechanism through inhibition of 5lipooxygenase and cyclooxygenase enzymes. Structure activity relationship study of these derivatives revealed that the styryl double bond is essential for 5-lipooxygenase inhibitory activities. Electron donating substituents on the phenyl ring lead to increase of 5lipooxygenase-inhibition. Compound (Ia) was the most active one of these derivatives, it inhibits carrageenan-induced edema by $37 \%$ at $20 \mathrm{mg} / \mathrm{kg}$ po. ${ }^{6}$ Guided by the aforementioned reports focusing on isoxazole derivatives as anti-inflammatory and the fact that isoxazole nucleus was incorporated in classical NSAIDs (isoxicam) ${ }^{7}$ selective $\mathrm{COX}-2$ inhibitors (valdecoxib), ${ }^{8}$ dual COX/5LO inhibitors and immunomodulatory agents (Leflunomide). ${ }^{9}$ The present work reports the design, synthesis and anti-inflammatory screening of some 5substitutedstyryl-isoxazole-3-carboxylic acid derivatives in ongoing efforts to develop a new potent and selective anti-inflammatory candidate with minimal GIT side effects.

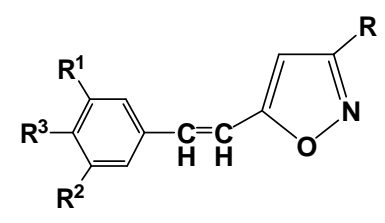

(I)

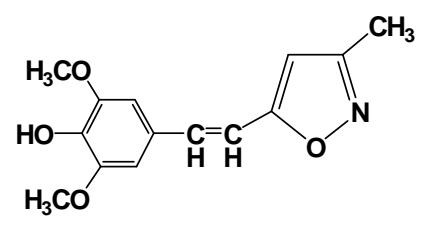

(Ia)
$\mathrm{R}=\mathrm{Me}, \mathrm{CF}_{3}$

$\mathrm{R}^{1}, \mathrm{R}^{2}=\mathrm{H}, \mathrm{MeO}, \mathrm{CH}_{3}, \mathrm{Cl}, \mathrm{Br}$, tert-Bu, iso-propyl

$\mathrm{R}^{3}=\mathrm{H}, \mathrm{OH}$

\section{MATERIALS AND METHODS}

\section{Chemistry}

Melting points were determined on electrothermal melting point apparatus and were uncorrected. Precoated Silica gel plates (Kiesel gel $0.25 \mathrm{~mm}$, 60G $\mathrm{F}_{254}$, Merck, Germany) were used for thin layer chromatography (TLC). IR spectra were recorded as $\mathrm{KBr}$ discs on a Shimadzu IR 20091527 spectrophotometer at the Faculty of Pharmacy, Assiut University, Assiut, Egypt. ${ }^{1}$ H-NMR spectra were measured on Varian Em- 
360L NMR Spectrophotometer $(60 \mathrm{MHz})$ (Varian, USA) at Faculty of Pharmacy, Assiut University, Assiut, Egypt; JNM-LA400 FT NMR system (400 MHz) (JEOL Co., Tokyo, Japan) at the central laboratory, Assiut University, Assiut, Egypt and a JNM-GX 400 FT NMR spectrophotometer (JEOL Co., Tokyo, Japan) at the Institute of Natural Medicine, Toyama Medical and Pharmaceutical University, Toyama, Japan. Chemical shifts were given in $\delta \mathrm{ppm}$ relative to tetramethylsilane (TMS). Elemental microanalyses were performed on Perkin-Elmer, 240 elemental analyzer, at the central laboratory, Assiut University, Perkin-EIMER 2400 elemental analyzer at the central laboratory, Cairo University. Anti-inflammatory activity was performed at the Department of Pharmacology, Faculty of Medicine, Assiut University, Assiut, Egypt. The stomach investigation was detected with a JEOL, JSM4500LV Scanning Electron Microscope at Central Laboratory, Assiut University, Assiut, Egypt. Indocid ${ }^{\circledR}$ capsule was donated by kahira Pharm. and Chem. Ind. Co., Cairo, Egypt. All chemicals and solvents are of reagent grades. Adult male albino rats were obtained from animal house of the faculty of Medicine, Assiut University.

\section{Synthesis of ethyl 2,4-dioxo-6-arylhex-5- enoate derivatives $(\mathbf{2 a - g})^{10}$}

The appropriate chalcone derivative (1a-g, 0.1 mole) was added portion wise to an icecooled suspension of sodium ethoxide $(6.8 \mathrm{~g}$, $0.1 \mathrm{~mole})$ in dry $\mathrm{n}$-hexane $(250 \mathrm{~mL})$. Dithyl oxalate (14.6 g, 0.1 mole) was then added dropwise to the resulting mixture and the mixture was further stirred at room temperature overnight. The separated product obtained after acidified with dilute sulphuric acid was filtered and recrystallized from ethanol. The physical and spectral data are listed in Table 1.

\section{Synthesis of 5-(p-substitutedstyryl)- isoxazole-3-carboxylic acid ethyl esters (3a- g) ${ }^{10}$ \\ Glacial acetic acid $(100 \mathrm{~mL})$ was added to solution of appropriate ethyl hex-5-enoate derivative (2a-g, 0.0033 mole) in ethanol (100}

$\mathrm{mL}$ ). A mixture of hydroxylamine hydrochloride $(0.5622 \mathrm{~g}, 0.0081 \mathrm{~mole})$, and sodium acetate $(0.5622 \mathrm{~g}, 0.0068 \mathrm{~mole})$ in water was added to the former one. The reaction mixture was refluxed for 2.25-4 hours, cooled to room temperature, extracted with chloroform, washed with water and dried with anhydrous sodium sulfate. Chloroform was removed under reduced pressure and the residue was crystallized from ethanol. The physical and spectral data are listed in Table 1.

\section{Synthesis of 5-(p-substitutedstyryl) isoxazole-3-carboxylic acids (4a-f) ${ }^{11}$ \\ Hydrochloric acid $(30 \mathrm{~mL})$ was added to the appropriate ethyl ester (3a-f, 0.0023 mole) dissolved in glacial acetic acid $(20 \mathrm{~mL})$. The mixture was refluxed for 1.5-5 hours and cooled to room temperature. The crystalline product, separated by filtration or extracted with chloroform/ether (60:40) and the organic layer was evaporated under reduced pressure, was recrystallized from suitable solvent. The physical and spectral data are listed in Table 1.}

\section{Synthesis of 5-(p-dimethylaminostyryl)- isoxazole-3-carboxylic acid (4g) ${ }^{11}$}

The ethyl ester $3 \mathrm{~g}$ ( $4.0 \mathrm{~g}, 0.014$ mole) was refluxed in hydrochloric acid $(30 \mathrm{~mL})$ for two hours. The reaction mixture was allowed to cool to room temperature. The separated crystalline product was filtered, washed with water and recrystallized from ethanol. The physical and spectral data are listed in Table 1.

\section{Synthesis of 5-(p-substitutedstyryl)- isoxazole-3-hydroxamic acids (5a-g) ${ }^{12}$}

The appropriate ethyl ester (3a-g, 0.004 mole) was refluxed in methanolic solution of hydroxylamine [prepared by neutralizing a solution of hydroxylamine hydrochloride (1.5 $\mathrm{g}, 0.022$ mole) in methanol with a solution of potassium hydroxide (1.4 g, 0.025 mole) in methanol] for 4 hours. The reaction mixture was concentrated under reduced pressure and the separated product was filtered and washed with water. The dried product was recrystallized from an appropriate solvent. The physical and spectral data are listed in Table 1. 
Table 1: The physical, IR and ${ }^{1} \mathrm{H}-\mathrm{NMR}$ data of the newly synthesized derivatives (2b,d; 3b,d; $\mathbf{4 b}$-g; 5a-g; 6-29).

\begin{tabular}{|c|c|c|c|c|c|c|c|c|c|}
\hline \multirow{2}{*}{$\begin{array}{l}\text { Compd. } \\
\text { no. }\end{array}$} & \multirow{2}{*}{$\begin{array}{l}\text { Yield } \\
(\%)\end{array}$} & \multirow{2}{*}{$\begin{array}{c}\text { M.P. } /{ }^{\circ} \mathrm{C} \\
\text { (Crystallization } \\
\text { Solvent) }\end{array}$} & \multicolumn{3}{|c|}{ IR ( $\mathrm{KBr}$ discs) $\mathrm{cm}^{-1}$} & \multirow{2}{*}{${ }^{1} \mathrm{H}-\mathrm{NMR} \delta$ ppm Chemical shift } & \multicolumn{3}{|c|}{$\begin{array}{l}\text { Microanalytical data (\%) } \\
\text { Cal./Found }\end{array}$} \\
\hline & & & $\mathrm{OH}$ & $\mathrm{NH}$ & $\mathrm{C}=\mathrm{O}$ & & $\mathrm{C}$ & $\mathrm{H}$ & $\mathrm{N}$ \\
\hline $2 \mathbf{b}^{\mathrm{a}^{* *}}$ & 49 & $\begin{array}{l}114-115 \\
\text { (Ehanol) }\end{array}$ & $\begin{array}{l}3430- \\
3535\end{array}$ & - & $\begin{array}{l}1722 \\
1631\end{array}$ & $\begin{array}{l}1.5\left(3 \mathrm{H}, \mathrm{t}, J=8 \mathrm{~Hz},-\mathrm{CH}_{2} \mathrm{CH}_{3}\right) ; 4.4(2 \mathrm{H}, \\
\left.\mathrm{q}, J=16 \mathrm{~Hz},-\mathrm{CH}_{2} \mathrm{CH}_{3}\right) ; 6.7(2 \mathrm{H}, \mathrm{s},- \\
\left.\mathrm{CH}_{2}\right) ; 6.8(\mathrm{H}, \mathrm{d}, J=16 \mathrm{~Hz}, \mathrm{H}-5) ; 7.4 \\
\left(2 \mathrm{H}, \mathrm{d}, J=8 \mathrm{~Hz}, \mathrm{H}-26^{\prime}\right) ; 7.8(2 \mathrm{H}, \mathrm{d}, J= \\
8 \mathrm{~Hz}, \mathrm{H}-3,5) ; 7.9(\mathrm{H}, \mathrm{d}, J=16 \mathrm{~Hz}, \mathrm{H}-6) .\end{array}$ & - & - & - \\
\hline $2 d^{\mathrm{a}^{* *}}$ & 62 & $\begin{array}{l}120-121 \\
\text { (Ethanol) }\end{array}$ & $\begin{array}{l}3420- \\
3555\end{array}$ & - & $\begin{array}{l}1715, \\
1627\end{array}$ & $\begin{array}{l}1.2\left(3 \mathrm{H}, \mathrm{t}, J=8 \mathrm{~Hz},-\mathrm{CH}_{2} \mathrm{CH}_{3}\right) ; 4.2(2 \mathrm{H}, \\
\left.\mathrm{q}, J=16 \mathrm{~Hz},-\mathrm{CH}_{2} \mathrm{CH}_{3}\right) ; 6.3(2 \mathrm{H}, \mathrm{s},- \\
\left.\mathrm{CH}_{2}\right) ; 6.5(\mathrm{H}, \mathrm{d}, J=16 \mathrm{~Hz}, \mathrm{H}-5) ; 7.3 \\
\left(5 \mathrm{H}, \mathrm{m}, \mathrm{H}-6 \text { and } 4-\mathrm{Br}_{-} \mathrm{C}_{6} \mathrm{H}_{4}\right)\end{array}$ & - & - & - \\
\hline $3 b^{a^{* *}}$ & 90 & $\begin{array}{l}140-141 \\
\text { (Ethanol) }\end{array}$ & - & - & 1725 & $\begin{array}{l}1.5\left(3 \mathrm{H}, \mathrm{t}, J=8 \mathrm{~Hz},-\mathrm{CH}_{2}-\mathrm{CH}_{3}\right) ; 4.6(2 \mathrm{H}, \\
\left.\mathrm{q}, J=14 \mathrm{~Hz},-\mathrm{CH}_{2}-\mathrm{CH}_{3}\right) ; 6.9\left(\mathrm{H}, \mathrm{s},-\mathrm{C}_{4} \mathrm{H}\right) ; \\
7.1\left(\mathrm{H}, \mathrm{d}, J=16 \mathrm{~Hz}-\mathrm{H}_{2} \text { vinyl }\right) ; 7.4-7.9 \\
\left(5 \mathrm{H}, \mathrm{m},-\mathrm{H}_{1} \text { vinyl and }-4-\mathrm{F}-\mathrm{C}_{6} \mathrm{H}_{4}\right) .\end{array}$ & $\begin{array}{l}64.36 \\
64.64\end{array}$ & $\begin{array}{l}4.63 \\
4.73\end{array}$ & $\begin{array}{l}5.36 \\
5.34\end{array}$ \\
\hline $3 d^{\mathrm{a}^{* *}}$ & 82 & $\begin{array}{l}143-144 \\
\text { (Ethanol) }\end{array}$ & - & - & 1727 & $\begin{array}{l}1.4\left(3 \mathrm{H}, \mathrm{t}, J=8 \mathrm{~Hz},-\mathrm{CH}_{2}-\mathrm{CH}_{3}\right) ; 4.4(2 \mathrm{H}, \\
\left.\mathrm{q}, J=14 \mathrm{~Hz},-\mathrm{CH}_{2}-\mathrm{CH}_{3}\right) ; 6.6\left(\mathrm{H}, \mathrm{s},-\mathrm{C}_{4} \mathrm{H}\right) ; \\
6.8\left(\mathrm{H}, \mathrm{d}, J=16 \mathrm{~Hz}-\mathrm{H}_{2} \text { vinyl }\right) ; 7.2-7.6 \\
\left(5 \mathrm{H}, \mathrm{m},-\mathrm{H}_{1} \text { vinyl and } 4-\mathrm{Br}_{-} \mathrm{C}_{6} \mathrm{H}_{4}\right) .\end{array}$ & $\begin{array}{l}54.22 \\
54.88\end{array}$ & $\begin{array}{c}3.9 \\
3.75\end{array}$ & $\begin{array}{c}4.5 \\
4.33\end{array}$ \\
\hline $4 b^{a^{*}}$ & 82 & $\begin{array}{l}\text { 202-203 } \\
\text { (Ethanol) }\end{array}$ & 3140 & - & 1703 & $\begin{array}{l}7.2\left(\mathrm{H}, \mathrm{s},-\mathrm{C}_{4} \mathrm{H}\right) ; 6.3-6.6\left(4 \mathrm{H}, \mathrm{m}, \mathrm{H}_{1}, \mathrm{H}_{2}\right. \\
\left.\text { vinyl and } \mathrm{H}-2^{\prime}, 6^{`}\right) ; 7.9(2 \mathrm{H}, \mathrm{d}, J=8 \mathrm{~Hz}, \\
\left.\mathrm{H}-3^{\prime}, 5^{\prime}\right) .\end{array}$ & $\begin{array}{l}61.80 \\
61.66\end{array}$ & $\begin{array}{l}3.46 \\
3.70\end{array}$ & $\begin{array}{l}6.01 \\
6.03\end{array}$ \\
\hline $4 c^{a^{*}}$ & 83 & $\begin{array}{c}220-222 \\
\text { (Ethanol) }\end{array}$ & 3140 & - & 1699 & $\begin{array}{l}7.2\left(\mathrm{H}, \mathrm{s},-\mathrm{C}_{4} \mathrm{H}\right) ; 7.5\left(\mathrm{H}, \mathrm{d}, J=16 \mathrm{~Hz},-\mathrm{H}_{2}\right. \\
\text { vinyl }) ; 7.7-8.1\left(5 \mathrm{H}, \mathrm{m}, \mathrm{H}_{1} \text { vinyl and 4- }\right. \\
\left.\mathrm{Cl}^{-} \mathrm{C}_{6} \mathrm{H}_{4}\right) .\end{array}$ & $\begin{array}{l}57.73 \\
57.84\end{array}$ & $\begin{array}{l}3.23 \\
3.65\end{array}$ & $\begin{array}{l}5.61 \\
5.62\end{array}$ \\
\hline $4 d^{\mathrm{a}^{*}}$ & 82 & $\begin{array}{l}224-226 \\
\text { (Ethanol) }\end{array}$ & 3175 & - & 1701 & 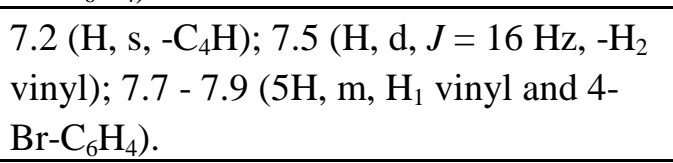 & $\begin{array}{l}49.01 \\
48.82\end{array}$ & $\begin{array}{l}2.74 \\
2.98\end{array}$ & $\begin{array}{l}4.76 \\
4.75\end{array}$ \\
\hline
\end{tabular}


Table 1: Continued

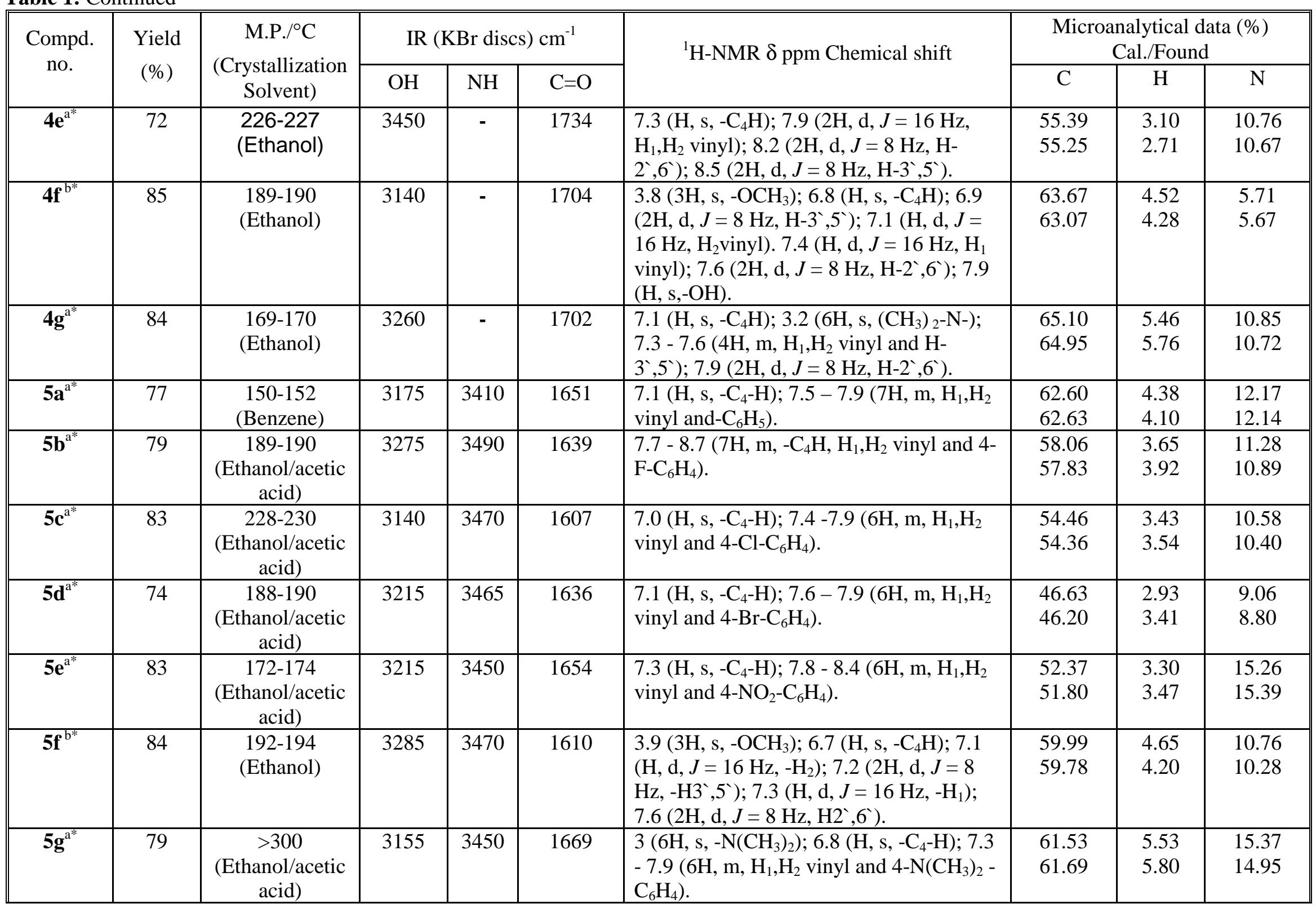


Table 1: Continued

\begin{tabular}{|c|c|c|c|c|c|c|c|c|c|}
\hline \multirow{2}{*}{$\begin{array}{l}\text { Compd. } \\
\text { no. }\end{array}$} & \multirow{2}{*}{$\begin{array}{l}\text { Yield } \\
(\%)\end{array}$} & \multirow{2}{*}{$\begin{array}{l}\text { M.P. } /{ }^{\circ} \mathrm{C} \\
\text { (Crystallization } \\
\text { Solvent) }\end{array}$} & \multicolumn{3}{|c|}{$\mathrm{IR}(\mathrm{KBr}$ discs $) \mathrm{cm}^{-1}$} & \multirow{2}{*}{${ }^{1} \mathrm{H}-\mathrm{NMR} \delta$ ppm Chemical shift } & \multicolumn{3}{|c|}{$\begin{array}{l}\text { Microanalytical data (\%) } \\
\text { Cal./Found }\end{array}$} \\
\hline & & & $\mathrm{OH}$ & $\mathrm{NH}$ & $\mathrm{C}=\mathrm{O}$ & & $\mathrm{C}$ & $\mathrm{H}$ & $\mathrm{N}$ \\
\hline $6^{\mathrm{a}^{*}}$ & 73 & $\begin{array}{c}163-164 \\
(\text { Ethanol) }\end{array}$ & - & 3330 & 1653 & $\begin{array}{l}2.9\left(3 \mathrm{H}, \mathrm{d}, J=6 \mathrm{~Hz},-\mathrm{CH}_{3}\right) ; 7.2(\mathrm{H}, \mathrm{s},- \\
\left.\mathrm{C}_{4} \mathrm{H}\right) ; 7.7-8.1(7 \mathrm{H}, \mathrm{m},-\mathrm{CH}=\mathrm{CH} \text { and } \\
\text { aromatic protons }) ; 9.0(\mathrm{H}, \mathrm{q}, J=6 \mathrm{~Hz},- \\
\mathrm{NH}) .\end{array}$ & $\begin{array}{l}68.40 \\
68.40\end{array}$ & $\begin{array}{l}5.29 \\
4.98\end{array}$ & $\begin{array}{l}12.27 \\
12.21\end{array}$ \\
\hline $7^{\mathrm{a}^{*}}$ & 80 & $\begin{array}{c}210-211 \\
\text { (Benzene) }\end{array}$ & - & 3360 & 1678 & $\begin{array}{l}3.0\left(3 \mathrm{H}, \mathrm{d}, J=5 \mathrm{~Hz},-\mathrm{CH}_{3}\right) ; 7.2(\mathrm{H}, \mathrm{s},- \\
\left.\mathrm{C}_{4} \mathrm{H}\right) ; 7.6-8.1(6 \mathrm{H}, \mathrm{m},-\mathrm{CH}=\mathrm{CH} \text { and } \\
\text { aromatic protons }) ; 9.0(\mathrm{H}, \mathrm{q}, J=5 \mathrm{~Hz},- \\
\mathrm{NH}) \text {. }\end{array}$ & $\begin{array}{l}59.44 \\
59.20\end{array}$ & $\begin{array}{l}4.22 \\
4.27\end{array}$ & $\begin{array}{l}10.66 \\
10.63\end{array}$ \\
\hline $8^{\mathrm{a}^{*}}$ & 57 & $\begin{array}{l}\text { 259-260 } \\
\text { (Ethanol/dimet } \\
\text { hylformamide) }\end{array}$ & - & 3350 & 1665 & $\begin{array}{l}3.0\left(3 \mathrm{H}, \mathrm{d}, J=4 \mathrm{~Hz},-\mathrm{CH}_{3}\right) ; 7.4(\mathrm{H}, \mathrm{s},- \\
\left.\mathrm{C}_{4} \mathrm{H}\right) ; 8.0-8.8(6 \mathrm{H}, \mathrm{m},-\mathrm{CH}=\mathrm{CH} \text { and } \\
\text { aromatic protons }) ; 9.1(\mathrm{H}, \mathrm{q}, J=4 \mathrm{~Hz},- \\
\mathrm{NH}) .\end{array}$ & $\begin{array}{l}57.14 \\
56.55\end{array}$ & $\begin{array}{l}4.05 \\
4.32\end{array}$ & $\begin{array}{l}15.38 \\
15.36\end{array}$ \\
\hline $9^{a^{*}}$ & 68 & $\begin{array}{l}133-135 \\
\text { (Ethanol) }\end{array}$ & - & 3350 & 1668 & $\begin{array}{l}2.9\left(3 \mathrm{H}, \mathrm{d}, J=4 \mathrm{~Hz},-\mathrm{CH}_{3}\right) ; 4(3 \mathrm{H}, \mathrm{s},- \\
\left.\mathrm{OCH}_{3}\right) ; 7.1\left(\mathrm{H}, \mathrm{s},-\mathrm{C}_{4}-\mathrm{H}\right) ; 7.2-7.9(6 \mathrm{H}, \\
\mathrm{m},-\mathrm{CH}=\mathrm{CH} \text { and aromatic protons }) ; 9.0 \\
(\mathrm{H}, \mathrm{q}, J=4 \mathrm{~Hz},-\mathrm{NH}) .\end{array}$ & $\begin{array}{l}65.11 \\
64.26\end{array}$ & $\begin{array}{l}5.46 \\
5.65\end{array}$ & $\begin{array}{l}10.85 \\
10.81\end{array}$ \\
\hline $10^{\mathrm{a}^{*}}$ & 57 & $\begin{array}{l}\text { 229-230 } \\
\text { (Ethanol/dimet } \\
\text { hylformamide) }\end{array}$ & - & 3335 & 1680 & $\begin{array}{l}7.3\left(\mathrm{H}, \mathrm{s},-\mathrm{C}_{4}-\mathrm{H}\right) ; 7.5-8.2(11 \mathrm{H}, \mathrm{m},- \\
\mathrm{CH}=\mathrm{CH} \text { and aromatic protons }) ; 11.1(\mathrm{H}, \\
\mathrm{s},-\mathrm{NH}) .\end{array}$ & $\begin{array}{l}66.57 \\
66.48\end{array}$ & $\begin{array}{l}4.03 \\
4.54\end{array}$ & $\begin{array}{l}8.62 \\
8.66\end{array}$ \\
\hline $11^{\mathrm{a}^{*}}$ & 73 & $\begin{array}{l}\text { 258-260 } \\
\text { (Ethanol/dimet } \\
\text { hylformamide) }\end{array}$ & - & 3330 & 1658 & $\begin{array}{l}7.4\left(\mathrm{H}, \mathrm{s},-\mathrm{C}_{4}-\mathrm{H}\right) ; 7.5-8.7(10 \mathrm{H}, \mathrm{m},- \\
\mathrm{CH}=\mathrm{CH} \text { and aromatic protons }) ; 11.2(\mathrm{H}, \\
\mathrm{s},-\mathrm{NH}) .\end{array}$ & $\begin{array}{l}64.47 \\
64.18\end{array}$ & $\begin{array}{l}3.91 \\
4.39\end{array}$ & $\begin{array}{l}12.53 \\
12.54\end{array}$ \\
\hline $12^{\mathrm{a}^{*}}$ & 78 & $\begin{array}{c}185-186 \\
\text { (Ethanol/dimet } \\
\text { hylformamide) }\end{array}$ & - & 3415 & 1670 & $\begin{array}{l}4.0\left(3 \mathrm{H}, \mathrm{s},-\mathrm{OCH}_{3}\right) ; 7.1\left(\mathrm{H}, \mathrm{s},-\mathrm{C}_{4}-\mathrm{H}\right) ; 7.2 \\
-8.3(11 \mathrm{H}, \mathrm{m},-\mathrm{CH}=\mathrm{CH} \text { and aromatic } \\
\text { protons }) ; 11.1(\mathrm{H}, \mathrm{s},-\mathrm{NH}) .\end{array}$ & $\begin{array}{l}71.24 \\
71.12\end{array}$ & $\begin{array}{l}5.03 \\
5.57\end{array}$ & $\begin{array}{l}8.74 \\
8.65\end{array}$ \\
\hline $13^{\mathrm{a}^{*}}$ & 60 & $\begin{array}{c}202-203 \\
\text { (Ethanol) }\end{array}$ & - & 3325 & 1680 & $\begin{array}{l}7.1\left(\mathrm{H}, \mathrm{s},-\mathrm{C}_{4}-\mathrm{H}\right) ; 7.2-8.1(11 \mathrm{H}, \mathrm{m},- \\
\mathrm{CH}=\mathrm{CH} \text { and aromatic protons }) ; 11.2(\mathrm{H}, \\
\mathrm{s},-\mathrm{NH}) .\end{array}$ & $\begin{array}{l}70.12 \\
69.73\end{array}$ & $\begin{array}{l}4.25 \\
4.25\end{array}$ & $\begin{array}{l}9.09 \\
9.09\end{array}$ \\
\hline
\end{tabular}


Table 1: Continued

\begin{tabular}{|c|c|c|c|c|c|c|c|c|c|}
\hline \multirow{2}{*}{$\begin{array}{l}\text { Compd. } \\
\text { no. }\end{array}$} & \multirow{2}{*}{$\begin{array}{l}\text { Yield } \\
(\%)\end{array}$} & \multirow{2}{*}{$\begin{array}{c}\text { M.P. } /{ }^{\circ} \mathrm{C} \\
\text { (Crystallization } \\
\text { Solvent) }\end{array}$} & \multicolumn{3}{|c|}{$\mathrm{IR}(\mathrm{KBr}$ discs $) \mathrm{cm}^{-1}$} & \multirow{2}{*}{${ }^{1} \mathrm{H}-\mathrm{NMR} \delta$ ppm Chemical shift } & \multicolumn{3}{|c|}{$\begin{array}{l}\text { Microanalytical data (\%) } \\
\text { Cal./Found }\end{array}$} \\
\hline & & & $\mathrm{OH}$ & $\mathrm{NH}$ & $\mathrm{C}=\mathrm{O}$ & & $\mathrm{C}$ & $\mathrm{H}$ & $\mathrm{N}$ \\
\hline $14^{\mathrm{a}^{*}}$ & 63 & $\begin{array}{c}224-226 \\
\text { (Ethanol) }\end{array}$ & - & 3340 & 1656 & $\begin{array}{l}7.2\left(\mathrm{H}, \mathrm{s},-\mathrm{C}_{4}-\mathrm{H}\right) ; 7.3-8.3(10 \mathrm{H}, \mathrm{m},- \\
\mathrm{CH}=\mathrm{CH} \text { and aromatic protons }) ; 11.2(\mathrm{H}, \\
\mathrm{s},-\mathrm{NH}) .\end{array}$ & $\begin{array}{l}63.08 \\
62.94\end{array}$ & $\begin{array}{l}3.53 \\
3.93\end{array}$ & $\begin{array}{l}8.17 \\
8.22\end{array}$ \\
\hline $15^{\mathrm{a}^{*}}$ & 72 & $\begin{array}{c}150-152 \\
\text { (Ethanol/dimet } \\
\text { hylformamide) }\end{array}$ & - & 3390 & 1662 & $\begin{array}{l}7.4\left(\mathrm{H}, \mathrm{s},-\mathrm{C}_{4}-\mathrm{H}\right) ; 7.5-8.7(10 \mathrm{H}, \mathrm{m},- \\
\mathrm{CH}=\mathrm{CH} \text { and aromatic protons }) ; 11.3(\mathrm{H}, \\
\mathrm{s},-\mathrm{NH})\end{array}$ & $\begin{array}{l}61.19 \\
61.19\end{array}$ & $\begin{array}{l}3.42 \\
3.49\end{array}$ & $\begin{array}{l}11.89 \\
11.96\end{array}$ \\
\hline $16^{\mathrm{a}^{*}}$ & 63 & $\begin{array}{l}240-241 \\
\text { (Ethanol) }\end{array}$ & - & 3390 & 1675 & $\begin{array}{l}4.0\left(3 \mathrm{H}, \mathrm{s},-\mathrm{OCH}_{3}\right) ; 7.1\left(\mathrm{H}, \mathrm{s},-\mathrm{C}_{4}-\mathrm{H}\right) ; 7.2 \\
-8.2(10 \mathrm{H}, \mathrm{m},-\mathrm{CH}=\mathrm{CH} \text { and aromatic } \\
\text { protons }) ; 11.2(\mathrm{H}, \mathrm{s},-\mathrm{NH}) .\end{array}$ & $\begin{array}{l}67.45 \\
67.10\end{array}$ & $\begin{array}{l}4.47 \\
4.53\end{array}$ & $\begin{array}{l}8.28 \\
8.34\end{array}$ \\
\hline $17^{\mathrm{a}^{*}}$ & 71 & $\begin{array}{c}212-214 \\
\text { (Ethanol/dimet } \\
\text { hylformamide) }\end{array}$ & - & 3335 & 1682 & $\begin{array}{l}7.4\left(\mathrm{H}, \mathrm{s},-\mathrm{C}_{4} \mathrm{H}\right) ; 7.7-8.3(11 \mathrm{H}, \mathrm{m},- \\
\mathrm{CH}=\mathrm{CH} \text { and aromatic protons); } 11.3(\mathrm{H}, \\
\text { s, }-\mathrm{NH}) \text {. }\end{array}$ & $\begin{array}{l}66.57 \\
66.34\end{array}$ & $\begin{array}{l}4.03 \\
4.40\end{array}$ & $\begin{array}{l}8.63 \\
8.64\end{array}$ \\
\hline $1^{\mathrm{a}^{\mathrm{a}^{*}}}$ & 69 & $\begin{array}{c}225-227 \\
\text { (Benzene/dimet } \\
\text { hylformamide) }\end{array}$ & - & 3390 & 1681 & $\begin{array}{l}7.4\left(\mathrm{H}, \mathrm{s},-\mathrm{C}_{4} \mathrm{H}\right) ; 7.6-8.3(10 \mathrm{H}, \mathrm{m},- \\
\mathrm{CH}=\mathrm{CH} \text { and aromatic protons }) ; 11.2(\mathrm{H}, \\
\mathrm{s},-\mathrm{NH}) .\end{array}$ & $\begin{array}{l}60.19 \\
60.23\end{array}$ & $\begin{array}{l}3.37 \\
3.76\end{array}$ & $\begin{array}{l}7.80 \\
7.84\end{array}$ \\
\hline $19^{a^{*}}$ & 67 & $\begin{array}{c}258-260 \\
\text { (Ethanol/dimet } \\
\text { hylformamide) }\end{array}$ & - & 3340 & 1662 & $\begin{array}{l}7.4\left(\mathrm{H}, \mathrm{s},-\mathrm{C}_{4} \mathrm{H}\right) ; 7.5-8.6(10 \mathrm{H}, \mathrm{m},- \\
\mathrm{CH}=\mathrm{CH} \text { and aromatic protons); } 11.1(\mathrm{H}, \\
\mathrm{s},-\mathrm{NH}) .\end{array}$ & $\begin{array}{l}58.47 \\
58.15\end{array}$ & $\begin{array}{l}3.27 \\
3.56\end{array}$ & $\begin{array}{l}11.36 \\
11.32\end{array}$ \\
\hline $\mathbf{2 0}^{\mathrm{a}^{*}}$ & 64 & $\begin{array}{c}\text { 204-206 } \\
\text { (Ethanol/dimet } \\
\text { hylformamide) }\end{array}$ & - & 3420 & 1676 & $\begin{array}{l}3.9\left(3 \mathrm{H}, \mathrm{s},-\mathrm{OCH}_{3}\right) ; 7.1\left(\mathrm{H}, \mathrm{s},-\mathrm{C}_{4}-\mathrm{H}\right) ; 7.2 \\
-8.4(10 \mathrm{H}, \mathrm{m},-\mathrm{CH}=\mathrm{CH} \text { and aromatic } \\
\text { protons }) ; 11.5(\mathrm{H}, \mathrm{s},-\mathrm{NH})\end{array}$ & $\begin{array}{l}64.32 \\
64.18\end{array}$ & $\begin{array}{l}4.26 \\
4.72\end{array}$ & $\begin{array}{l}7.89 \\
7.77\end{array}$ \\
\hline $21^{\mathrm{a}^{*}}$ & 57 & $\begin{array}{c}265-266 \\
\text { (Ethanol/dimet } \\
\text { hylformamide) }\end{array}$ & - & $\begin{array}{c}3500 \\
- \\
3400\end{array}$ & 1670 & $\begin{array}{l}7.4\left(\mathrm{H}, \mathrm{s},-\mathrm{C}_{4} \mathrm{H}\right) ; 7.6-8.1(9 \mathrm{H}, \mathrm{m},- \\
\mathrm{CH}=\mathrm{CH} \text { and aromatic protons). }\end{array}$ & $\begin{array}{l}60.59 \\
60.34\end{array}$ & $\begin{array}{l}3.73 \\
3.31\end{array}$ & $\begin{array}{l}14.13 \\
13.90\end{array}$ \\
\hline $\mathbf{2 2}^{\mathrm{a}^{*}}$ & 61 & $\begin{array}{c}218-220 \\
\text { (Ethanol/dimet } \\
\text { hylformamide) }\end{array}$ & - & $\begin{array}{c}3550 \\
- \\
3400\end{array}$ & 1661 & $\begin{array}{l}7.6\left(\mathrm{H}, \mathrm{s},-\mathrm{C}_{4}-\mathrm{H}\right) ; 7.7-8.6(8 \mathrm{H}, \mathrm{m},- \\
\mathrm{CH}=\mathrm{CH} \text { and aromatic protons }) ; 10.5(\mathrm{H}, \\
\mathrm{s}, \mathrm{NH}) .\end{array}$ & $\begin{array}{l}57.14 \\
57.45\end{array}$ & $\begin{array}{l}3.20 \\
3.12\end{array}$ & $\begin{array}{l}13.33 \\
13.22\end{array}$ \\
\hline $\mathbf{2 3}^{\mathrm{a}^{*}}$ & 67 & $\begin{array}{c}274-275 \\
\text { (Ethanol/dimet } \\
\text { hylformamide) }\end{array}$ & - & $\begin{array}{c}3550 \\
- \\
3375\end{array}$ & 1656 & $\begin{array}{l}7.3\left(\mathrm{H}, \mathrm{s},-\mathrm{C}_{4}-\mathrm{H}\right) ; 7.5-8.0(8 \mathrm{H}, \mathrm{m},- \\
\mathrm{CH}=\mathrm{CH} \text { and aromatic protons }) .\end{array}$ & $\begin{array}{l}54.30 \\
54.42\end{array}$ & $\begin{array}{l}3.04 \\
2.92\end{array}$ & $\begin{array}{l}12.66 \\
12.44\end{array}$ \\
\hline
\end{tabular}


Table 1: Continued

\begin{tabular}{|c|c|c|c|c|c|c|c|c|c|}
\hline \multirow{2}{*}{$\begin{array}{l}\text { Compd. } \\
\text { no. }\end{array}$} & \multirow{2}{*}{$\begin{array}{l}\text { Yield } \\
(\%)\end{array}$} & \multirow{2}{*}{$\begin{array}{l}\text { M.P. }{ }^{\circ} \mathrm{C} \\
\text { (Crystallization } \\
\text { Solvent) }\end{array}$} & \multicolumn{3}{|c|}{$\mathrm{IR}(\mathrm{KBr}$ discs $) \mathrm{cm}^{-1}$} & \multirow{2}{*}{${ }^{1} \mathrm{H}-\mathrm{NMR} \delta$ ppm Chemical shift } & \multicolumn{3}{|c|}{$\begin{array}{l}\text { Microanalytical data }(\%) \\
\text { Cal./Found }\end{array}$} \\
\hline & & & $\mathrm{OH}$ & $\mathrm{NH}$ & $\mathrm{C}=\mathrm{O}$ & & $\mathrm{C}$ & $\mathrm{H}$ & $\mathrm{N}$ \\
\hline $24^{\mathrm{a}^{*}}$ & 65 & $\begin{array}{l}\text { 206-208 } \\
\text { (Ethanol/dimet } \\
\text { hylformamide) }\end{array}$ & - & $\begin{array}{c}3550 \\
- \\
3480\end{array}$ & 1667 & $\begin{array}{l}7.2\left(\mathrm{H}, \mathrm{s},-\mathrm{C}_{4} \mathrm{H}\right) ; 7.6-8.6(8 \mathrm{H}, \mathrm{m},- \\
\mathrm{CH}=\mathrm{CH} \text { and aromatic protons }) .\end{array}$ & $\begin{array}{l}52.63 \\
52.97\end{array}$ & $\begin{array}{l}2.94 \\
2.78\end{array}$ & $\begin{array}{l}16.37 \\
16.14\end{array}$ \\
\hline $25^{\mathrm{a}^{*}}$ & 63 & $\begin{array}{c}201-202 \\
(\text { Ethanol })\end{array}$ & - & $\begin{array}{c}3510 \\
- \\
3390\end{array}$ & 1672 & $\begin{array}{l}4.0\left(3 \mathrm{H}, \mathrm{s},-\mathrm{OCH}_{3}\right) ; 7.0-8.8(9 \mathrm{H}, \mathrm{m},- \\
\mathrm{CH}=\mathrm{CH} \text { and aromatic protons }) .\end{array}$ & $\begin{array}{l}58.70 \\
58.83\end{array}$ & $\begin{array}{c}4.0 \\
3.75\end{array}$ & $\begin{array}{l}12.84 \\
12.98\end{array}$ \\
\hline $26^{\mathrm{a}^{*}}$ & 61 & $\begin{array}{l}\text { 150-152 } \\
\text { (Benzene/dimet } \\
\text { hylformamide }\end{array}$ & - & 3310 & 1648 & $\begin{array}{l}1.1-2.0\left(11 \mathrm{H}, \mathrm{m},-\mathrm{C}_{6} \mathrm{H}_{11}\right) ; 7.0(\mathrm{H}, \mathrm{s},- \\
\left.\mathrm{C}_{4} \mathrm{H}\right) ; 7.5-7.9(7 \mathrm{H}, \mathrm{m},-\mathrm{CH}=\mathrm{CH} \text { and } \\
\text { aromatic protons }) ; 8.3(\mathrm{H}, \mathrm{d}, J=6 \mathrm{~Hz},- \\
\mathrm{NH}) \text {. }\end{array}$ & $\begin{array}{l}72.95 \\
72.69\end{array}$ & $\begin{array}{c}6.8 \\
6.58\end{array}$ & $\begin{array}{l}9.45 \\
9.49\end{array}$ \\
\hline $27^{\mathrm{a}^{*}}$ & 66 & $\begin{array}{c}222-224 \\
\text { (Benzene) }\end{array}$ & - & 3315 & 1641 & $\begin{array}{l}1.2-2.0\left(11 \mathrm{H}, \mathrm{m},-\mathrm{C}_{6} \mathrm{H}_{11}\right) ; 7.0(\mathrm{H}, \mathrm{s},- \\
\left.\mathrm{C}_{4} \mathrm{H}\right) ; 7.3-7.8(6 \mathrm{H}, \mathrm{m},-\mathrm{CH}=\mathrm{CH} \text { and } \\
\text { aromatic protons }) ; 8.3(\mathrm{H}, \mathrm{d}, J=7 \mathrm{~Hz},- \\
\mathrm{NH}) .\end{array}$ & $\begin{array}{l}65.35 \\
65.07\end{array}$ & $\begin{array}{l}5.79 \\
6.51\end{array}$ & $\begin{array}{l}8.47 \\
8.48\end{array}$ \\
\hline $28^{\mathrm{a}^{*}}$ & 68 & $\begin{array}{l}\text { 249-250 } \\
\text { (Ethanol/dimet } \\
\text { hylformamide) }\end{array}$ & - & 3315 & 1645 & $\begin{array}{l}1.2-1.9\left(11 \mathrm{H}, \mathrm{m},-\mathrm{C}_{6} \mathrm{H}_{11}\right) ; 7.1(\mathrm{H}, \mathrm{s},- \\
\left.\mathrm{C}_{4} \mathrm{H}\right) ; 7.6-8.4(6 \mathrm{H}, \mathrm{m},-\mathrm{CH}=\mathrm{CH} \text { and } \\
\text { aromatic protons }) ; 8.5(\mathrm{H}, \mathrm{d}, J=5 \mathrm{~Hz},- \\
\mathrm{NH}) \text {. }\end{array}$ & $\begin{array}{l}63.33 \\
63.32\end{array}$ & $\begin{array}{l}5.61 \\
5.50\end{array}$ & $\begin{array}{l}12.31 \\
12.32\end{array}$ \\
\hline $29^{\mathrm{a}^{*}}$ & 61 & $\begin{array}{c}216-217 \\
\text { (Benzene) }\end{array}$ & - & 3320 & 1646 & $\begin{array}{l}\text { 1.4-1.9 }\left(11 \mathrm{H}, \mathrm{m},-\mathrm{C}_{6} \mathrm{H}_{11}\right) ; 4(3 \mathrm{H}, \mathrm{s},- \\
\left.\mathrm{OCH}_{3}\right) ; 7.1\left(\mathrm{H}, \mathrm{s},-\mathrm{C}_{4}-\mathrm{H}\right) 7.2-8.0(6 \mathrm{H}, \mathrm{m}, \\
-\mathrm{CH}=\mathrm{CH} \text { and aromatic protons }) ; 8.7(\mathrm{H}, \\
\mathrm{d}, J=7 \mathrm{~Hz},-\mathrm{NH}) .\end{array}$ & $\begin{array}{l}69.91 \\
69.90\end{array}$ & $\begin{array}{l}6.79 \\
6.67\end{array}$ & $\begin{array}{l}8.58 \\
8.46\end{array}$ \\
\hline
\end{tabular}

a- $60 \mathrm{MHz}$ b- $400 \mathrm{MHz} * \mathrm{DMSO}$ as solvent $* * \mathrm{CDCl}_{3}$ as solvent. 
General procedure for synthesis of 5-(psubstitutedstyryl)-isoxazole-3-carboxamides (6-29)

The appropriate acid (0.002 mole) was refluxed for 3 hours in excess thionyl chloride $(30 \mathrm{~mL})$. The corresponding amine (0.005 mole) in benzene was added dropwise to the residue obtained after evaporation of excess thionyl chloride under reduced pressure. The mixture was refluxed for 2-4 hours and the separated product obtained after concentration under reduced pressure was filtered, washed with dilute hydrochloric acid followed by water, dried and recrystallized from a suitable solvent. The physical and spectral data are listed in Table 1.

\section{Biological investigations}

\section{1- Anti-inflammatory activity}

The anti-inflammatory activity of twentyone representatives of synthesized compounds [(3a,e,f and g); (4a-g); (5a-g); 22; 24 and 25] was evaluated in rats by the carrageenaninduced edema method as described by Nargund et al. ${ }^{13}$ in comparison to indomethacin as a reference drug. Adult male albino rats weighing (120-150 g) were divided into groups, each of four animals. Solution or suspension of the test compound or reference drug in $6 \%$ tween 80 was administrated orally into rats at dose level of $100 \mathrm{mg} / \mathrm{kg}$. One group of animals was used for each treatment. Control animals were similarly treated with $6 \%$ tween 80 . After 30 minutes $0.1 \mathrm{~mL}$ of freshly prepared $1 \%$ carrageenan solution in normal saline was injected into the subplantar region of the right hind paw. The right paw volume was measured by a Veriner caliper (SMIEC) directly before and at one hour intervals after administration of the test compound for five hours. The antiinflammatory activity of the tested compound and reference drug was determined with the following formula

$\%$ Inhibition of inflammation $=\left(1-\mathrm{V}_{\mathrm{t}} / \mathrm{V}_{\mathrm{o}}\right) \times 100$ where $V_{t}, V_{o}$ represents the mean increase in paw volume in rats treated with tested compounds, control rats respectively.

The results of anti-inflammatory evaluation of the test compounds and reference drug are listed in Table 2.

\section{2- Ulcerogenicity ${ }^{14}$}

On the basis of preliminary results, three compounds $(\mathbf{3 g}, \mathbf{4 g}, \mathbf{5 g})$ were subjected to this investigation. Male adult albino rats (120-150 g) were divided into groups each of four animals. Animals were starved but had free access to water 24 hours prior to administration of drug. The animals were then treated orally by mean of stomach tube with solution or suspension of tested compounds and indomethacin as reference drug in tween 80 $(6 \%)$ at a dose level $100 \mathrm{mg} / \mathrm{kg}$. Control animals were treated with an equal volume of tween 80. Food was withdrawn from all groups until 24 hours after administration of drug. The rats were then sacrificed, so that the stomach could be removed, open along the greater curvature and clean gently by dipping in saline. Randomly selected specimens were then taken and prepared for scanning in an electron microscope. Specimens were fixed by soaking in glutaraldehyde solution $(5 \%$ in cacodylate buffer; $\mathrm{pH}$ 7.2) for 24 hours followed by three washing each for 20 minutes with cacodylate buffer. The specimens then treated with osmium tetraoxide (1\% solution) for 2 hours and washed with cacodylate buffer as shown above. The specimens were then subjected to dehydration by treatment for 30 minutes with each of 30\%, 50\% and 70\% ethanolic solution followed by $90 \%$ ethanol for one hour and finally in absolute ethanol for two days. After discharge of alcohol the specimens were soaked in amyl acetate solution for two days, dried under reduced pressure, mounted on holder and coated for scanning in a scanning electron microscope (SEM).

\section{RESULTS AND DISCUSSION}

\section{Chemistry}

The synthetic pathways for the preparation of the target derivatives are outlined in Scheme 1. In the present work, the $3+2$ route has been adopted for synthesis of the targeted isoxazole derivatives. The required three atomic component synthons were represented by 1,3 dicarbonyl system substituted by a styryl moiety which is intended to be located at C-5 of the isoxazole nucleus. Meanwhile, the planned carboxyl functionality at C-3 of the ring was introduced to the 1,3-dicarbonyl structures through interaction with diethyloxalate. The 1,3-dicarbonyl systems [2,4-dioxo-6-arylhex-5-enoic acid ethyl esters (2a-g)] acts as key 
Table 2: Anti-inflammatory activity of 5-[2-(p-substitiutedphenyl)vinyl]isoxazole-3-carboxylic acid derivatives (3b, 3e, 3f, 3g, 4a-g, 5a-g, 22, 24 and 25).<smiles>[R]C(=O)c1cc(C=Cc2ccc([R])cc2)on1</smiles>

\begin{tabular}{|c|c|c|c|c|c|c|c|}
\hline \multirow[t]{2}{*}{ Compd. No. } & \multirow[t]{2}{*}{$\mathrm{R}$} & \multirow[t]{2}{*}{$\mathrm{R}^{\prime}$} & \multicolumn{5}{|c|}{$\begin{array}{l}\text { Anti-inflammatory activity }(100 \mathrm{mg} / \mathrm{Kg}, \mathrm{p} . \mathrm{o}) \\
\text { \%Inhibition* }\end{array}$} \\
\hline & & & $1 \mathrm{hr}$ & $2 \mathrm{hr}$ & $3 \mathrm{hr}$ & $4 \mathrm{hr}$ & $5 \mathrm{hr}$ \\
\hline Indomethacin & & & 62.5 & 75 & 75 & 77.7 & 88.8 \\
\hline $3 \mathbf{b}$ & $\mathrm{F}$ & $\mathrm{OEt}$ & 37 & 50 & 55 & 68 & 72 \\
\hline $3 e$ & $\mathrm{NO}_{2}$ & $\mathrm{OEt}$ & 37 & 66 & 88 & 90 & 90 \\
\hline $3 f$ & $\mathrm{OCH}_{3}$ & $\mathrm{OEt}$ & 43 & 44 & 55 & 63 & 68 \\
\hline $3 g$ & $\mathrm{~N}\left(\mathrm{CH}_{3}\right)_{2}$ & $\mathrm{OEt}$ & 50 & 61 & 72 & 96 & 90 \\
\hline $4 a$ & $\mathrm{H}$ & $\mathrm{OH}$ & 61.25 & 68.75 & 37.5 & 33 & 33.3 \\
\hline $4 b$ & $\mathrm{~F}$ & $\mathrm{OH}$ & 75 & 75 & 75 & 72.5 & 81.5 \\
\hline $4 c$ & $\mathrm{Cl}$ & $\mathrm{OH}$ & 50 & 50 & 37.5 & 44.4 & 44.4 \\
\hline $4 d$ & $\mathrm{Br}$ & $\mathrm{OH}$ & 62.5 & 50 & 37.5 & 55.5 & 55.5 \\
\hline $4 e$ & $\mathrm{NO}_{2}$ & $\mathrm{OH}$ & 75 & 62.5 & 62.5 & 66.6 & 55.5 \\
\hline $4 f$ & $\mathrm{OCH}_{3}$ & $\mathrm{OH}$ & 75 & 25 & 33.3 & 22 & 22.2 \\
\hline $4 g$ & $\mathrm{~N}(\mathrm{CH} 3)_{2}$ & $\mathrm{OH}$ & 62.5 & 75 & 75 & 77.7 & 77.7 \\
\hline $5 a$ & $\mathrm{H}$ & $\mathrm{NHOH}$ & 33.3 & 50 & 37.25 & 55.5 & 66.6 \\
\hline $5 b$ & $\mathrm{~F}$ & $\mathrm{NHOH}$ & 62.25 & 62.5 & 62.5 & 22.2 & 22.2 \\
\hline $5 c$ & $\mathrm{Cl}$ & $\mathrm{NHOH}$ & 31.25 & 31.25 & 43.75 & 27.7 & 38.8 \\
\hline $5 d$ & $\mathrm{Br}$ & $\mathrm{NHOH}$ & 50 & 50 & 37.5 & 44.4 & 44.4 \\
\hline $5 e$ & $\mathrm{NO}_{2}$ & $\mathrm{NHOH}$ & 50 & 37.5 & 50 & 55.5 & 55.5 \\
\hline $5 f$ & $\mathrm{OCH}_{3}$ & $\mathrm{NHOH}$ & 75 & 62.5 & 62.5 & 73.3 & 73.3 \\
\hline $5 \mathrm{~g}$ & $\mathrm{~N}\left(\mathrm{CH}_{3}\right)_{2}$ & $\mathrm{NHOH}$ & 62.5 & 75 & 75 & 66.6 & 66.6 \\
\hline 22 & $\mathrm{~F}$ & NH-thiazolyl & 25 & 33 & 33 & 63 & 54.5 \\
\hline 24 & $\mathrm{NO}_{2}$ & NH-thiazolyl & 37.5 & 44 & 44 & 54.5 & 36 \\
\hline 25 & $\mathrm{OCH}_{3}$ & NH-thiazolyl & 12.5 & 44 & 44 & 54.5 & 63 \\
\hline
\end{tabular}

*All results are significantly different from control at $\mathrm{p}<0.005$. 


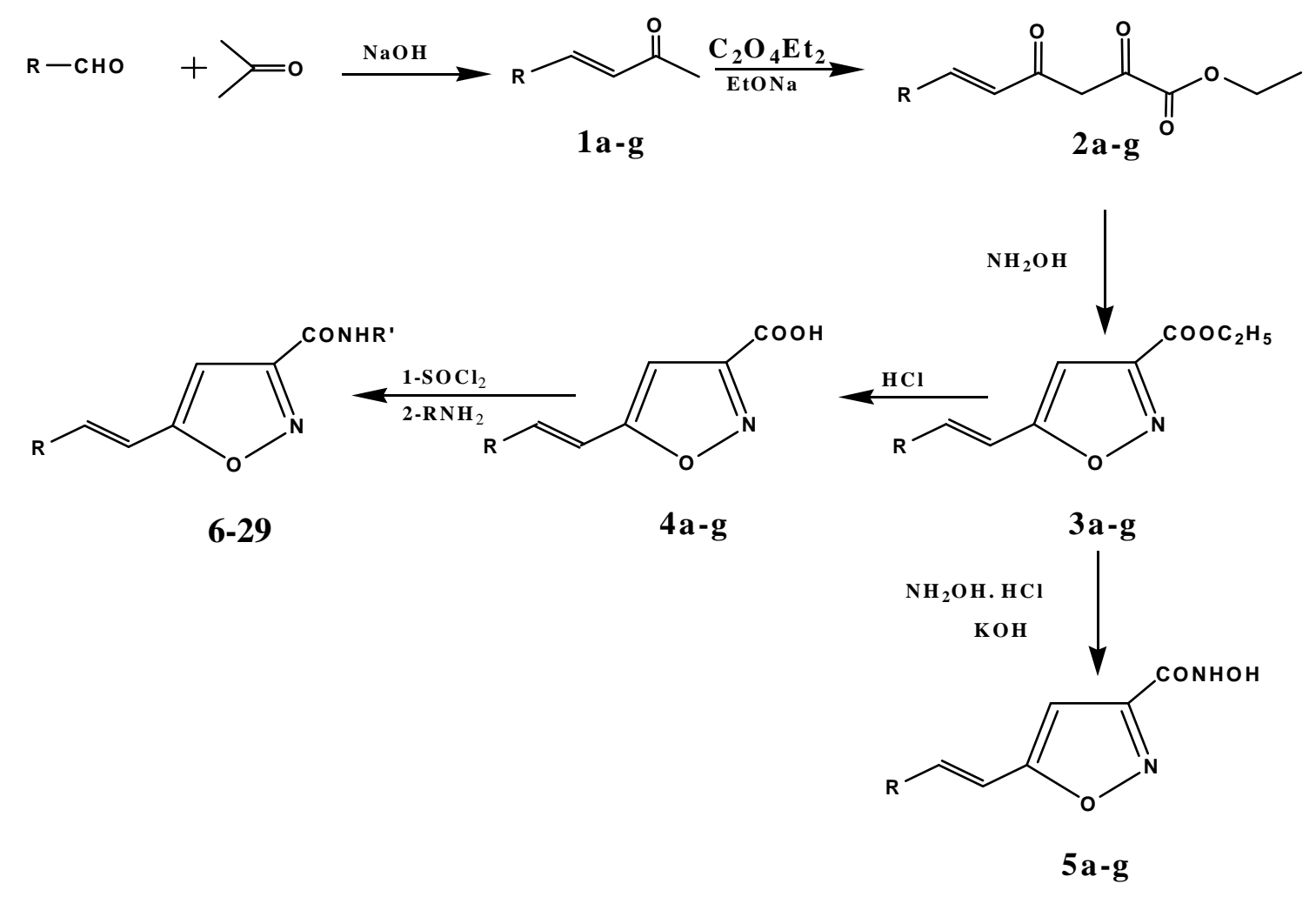

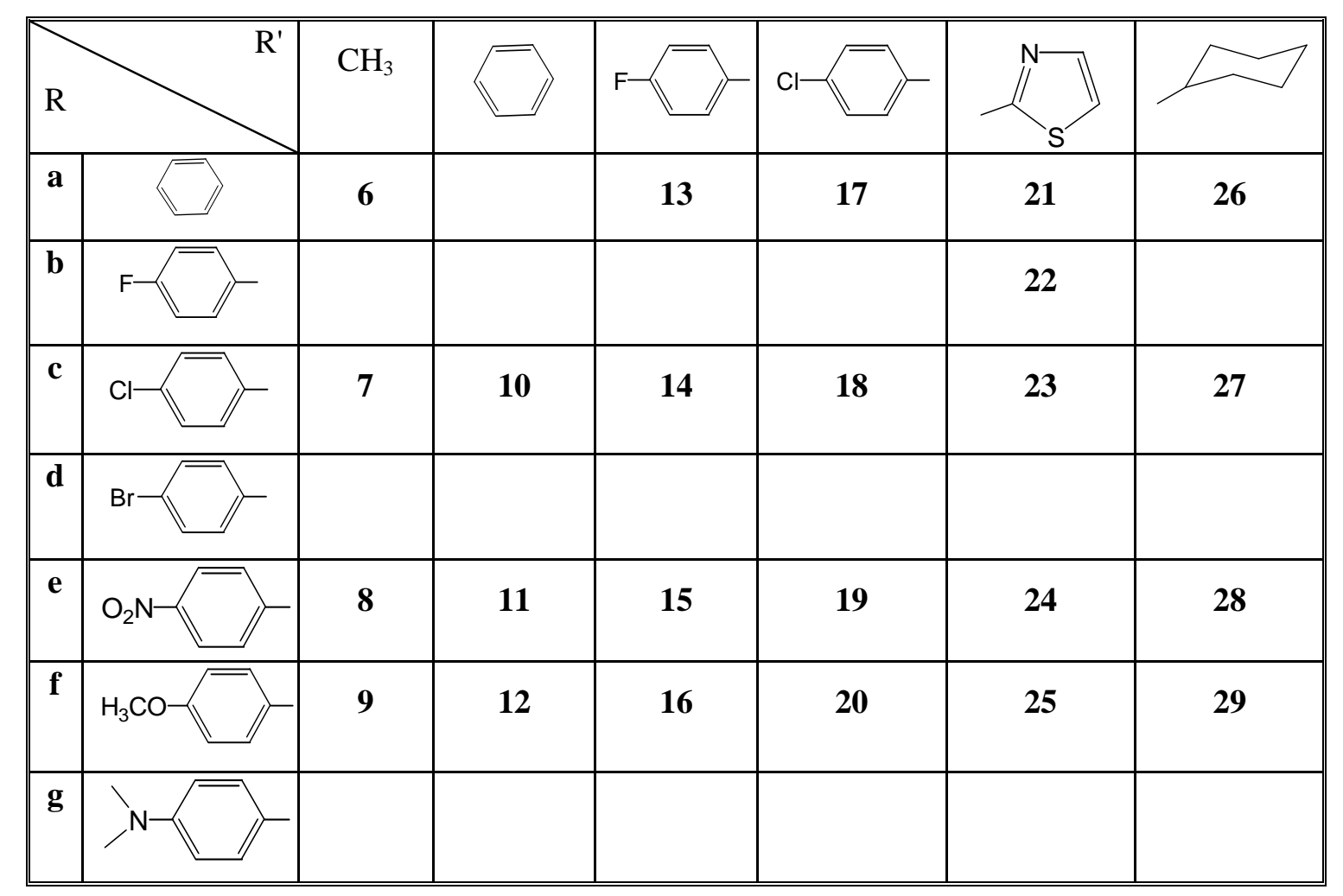

Scheme 1: Pathway for the synthesis of the target compounds 
intermediate for synthesis of the targeted 5(substituted) isoxazole-3-carboxylic acid derivatives. The synthesis of these intermediate was achieved through the reaction of chalcones (1a-g), prepared via aldol condensation of the respective substituted benzaldehyde with acetone in alkaline medium, with diethyl oxalate. The 2,4-diketoesters 2a-h were condensed with hydroxylamine hydrochloride in presence of acetic acid/sodium acetate, to afford mainly the targeted 5-( $p$ substitutedstyryl)-isoxazole-3-carboxylic acid esters 3a-h in good yields. The regiospecificity of this reaction could be explained on basis of reported observations. ${ }^{15}$ It is evident that the ethoxycarbonyl moiety enhances the electrophilicity of the neighboring C-atom of the 2,4-diketoester and render it preferentially susceptible for attack by $\mathrm{NH}_{2} \mathrm{OH}$. Moreover, control of the $\mathrm{pH}$ of the reaction through the use acetic acid/sodium acetate buffer $(\mathrm{pH} 4)$ instead of acetic acid only renders hydroxylamine in the $\mathrm{H}_{2} \mathrm{NOH}$ form. In this case the nitrogen atom is more nucleophilic and will attack the most electrophilic C-atom $\left(\mathrm{C}_{2}=\mathrm{O}\right)$. The suggested sequence of the condensationcyclization reaction involved in the formation of the targeted 5-(p-substitutedstyryl)isoxazole-3-carboxylic acid esters $\mathbf{3 a - h}$ can be illustrated by Chart 1 .

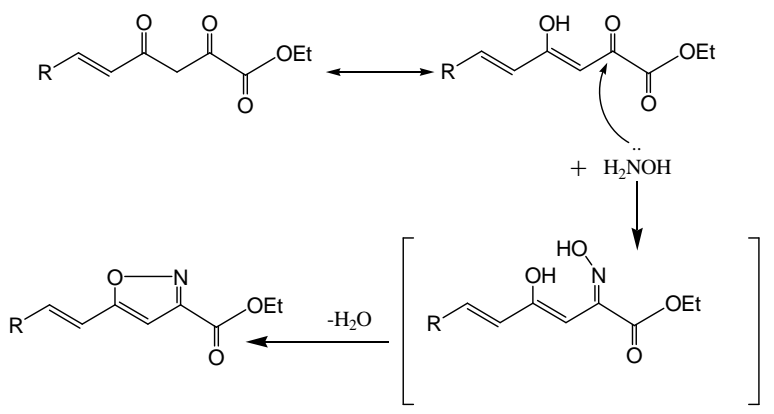

Chart 1

The resulting 5-(p-substitutedstyryl)isoxazole-3-carboxylic acid esters 3a-h were then converted to the corresponding acids $\mathbf{4 a - h}$ by boiling with hydrochloric acid. ${ }^{16}$ In this work dissolution of the isoxazole esters $\mathbf{3 a - h}$ in acetic acid followed by boiling in hydrochloric acid for 2 hours afforded the isoxazole carboxylic acids $\mathbf{4 a - h}$ in good yields. The physical and spectral data of the prepared compounds are agreed with reported data and that for the newly synthesized derivatives are mentioned in Table 1. The purity of the synthesized derivatives was monitored by TLC and by using microanalysis for the newly synthesized targets in addition to that compounds subjected to anti-inflammatory evaluation. The IR spectra of the esters 3a-h revealed the characteristic $\mathrm{C}=\mathrm{O}$ at $\delta 1730-1710$ $\mathrm{cm}^{-1}$, the vinyl $\mathrm{C}=\mathrm{C}$ at $\delta 1648-1621 \mathrm{~cm}^{-1}$ and the ester C-O-C at $\delta 1293-1201 \mathrm{~cm}^{-1}$. The ${ }^{1} \mathrm{H}-\mathrm{NMR}$ spectral data of the esters revealed the characteristic singlet at $\delta 6.55-6.90 \mathrm{ppm}$ that could be assigned to $\mathrm{C}_{4}-\mathrm{H}$ of the isoxazole nucleus. This assignment is based on comparison to reported value of similar compounds. ${ }^{17-20}$ On other hand the $J$ constant value of the vinyl protons support the suggested trans configuration of these protons. The vinyl and aryl protons revealed similar splitting and chemical shift as hexenoates $\mathbf{2 a - h}$. Further structural elucidation of the formed isoxazole derivatives has been achieved through ${ }^{13} \mathrm{C}-\mathrm{NMR}$ spectroscopy $\mathbf{3 g}$ as representative of this series. The spectrum revealed the characteristic chemical shifts of isoxazole C-atoms, $\delta$ C-4 (99.73 ppm), $\delta$ C-5 (156.57 ppm), $\delta \mathrm{C}-3$ (160.26 ppm). In addition to ester $\delta \mathrm{C}=\mathrm{O}(171.47 \mathrm{ppm})$; the $\mathrm{CH}_{2} \mathrm{CH}_{3}$ signals at $\delta=14.14$ and 61.98 respectively; dimethylaminocarbons $(\delta=40.15)$. The vinyl C-atoms appeared at $\delta=107.46$ for $\mathrm{C}_{1}, \delta=$ $136.46 \mathrm{ppm}$ for $\mathrm{C}_{2}$. The phenyl C-atoms appeared at $\delta=112.02$ ppm for $C_{2,6}$, at $\delta=$ $128.69 \mathrm{ppm}$ for $\mathrm{C}_{3,5}$. The assignment of the observed chemical shifts to the respective Catoms is based on comparison with reported value for the isoxazole nucleus, ${ }^{21}$ as well as other related compounds such as ethyl 5-[2-(2pyridyl)vinyl]isoxazole-3-carboxylate. ${ }^{20}$ The mass spectra of the compound $\mathbf{3 g}$ was agreed with the fact that isoxazole fragmented via formation azirine intermediate, revealed the molecular ion beak at $\mathrm{m} / \mathrm{z} 286$, and $\mathrm{M}^{-1}$ at $\mathrm{m} / \mathrm{z}$ 285 (base peak).

The economical procedure for preparation of hydroxamic acid derivatives is the reaction of hydroxylamine hydrochloride with acid chloride or esters. In this work, the isoxazole hydroxamic acid derivatives (5a-g) were prepared, according to scheme 1 taking the general procedure as a guide, by refluxing the appropriate ester (3a-g) with hydroxylamine in slightly alkaline $\mathrm{pH}$ for 3-4 hours resulted in hydroxamic acids (5a-g) in a good yield. The reaction of esters (3a-g) with amines were failed to give amide and the ester was recovered unchanged where the amide 
derivatives 6-29 were prepared from acid chloride. Reaction of acid chloride with solution or suspension of appropriate amine in benzene was give amide (6-29) in good yield. The synthesized derivatives were characterized through elemental analyses and their structures were confirmed by spectral data.

\section{Biological investigations}

5-( $p$-Substitutedstyryl)-isoxazole-3carboxylic acid (4a-g), hydroxamic acid (5a-h), carboxylic acid ethyl ester (3b,e-g) and carboxylic acid amide $(\mathbf{2 2}, \mathbf{2 4}, \mathbf{2 5})$ derivatives were tested for their anti-inflammatory activity. The anti-inflammatory marker was the percentage inhibition of carrageenan-induced rat paw edema. The tested compounds (100 $\mathrm{mg} / \mathrm{kg}$ ) were given orally, and then half an hour later, an edema was induced by subcutaneous injection of carrageenan into paw pad of the rat. Table 2 shows the results (\% inhibition) of anti-inflammatory activity of the tested compounds at one-hour time interval for five hours. p-Flourophenyl and pdimethylaminostyryl isoxazole carboxylic acid derivatives (4b and $\mathbf{4 g}$ ) showed maximum inhibition of inflammation ranging from $62 \sim 81.5 \%$. The inhibitory effect of inflammation of $\mathbf{4 b}$ and $\mathbf{4 g}$ starts after $1 \mathrm{hr}$ and maintained or slightly increased up to $5 \mathrm{hr}$. On the other hand, p-nitrophenyl and pmethoxystyryl isoxazole carboxylic acid derivatives $(\mathbf{4 e}$ and $\mathbf{4 f})$ revealed $75 \%$ inhibition of inflammation after $1 \mathrm{hr}$, which by time slightly decreased, as in case of $\mathbf{4 e}$, or dramatically decreased, as in case of $\mathbf{4 f}$. Other carboxylic acid derivatives (4a, $\mathbf{4 c}$ and $\mathbf{4 d}$ ) showed significant inhibitory activity (33 68\%) but less than reference drug, indomethacin (62 88.8) (Fig. 1).

In the group of isoxazole hydroxamic acid derivatives (5a-g), p-methoxyphenylderivative (5f) revealed $75 \%$ inhibition of inflammation after $1 \mathrm{hr}$, while other hydroxamic acid derivatives (5a-e and $\mathbf{5 g}$ ) showed inferior activity than indomethacin (Fig. 2).

Maximum inhibitory activity of inflammation was observed in isoxazole carboxylic acid ethyl esters (3e and $\mathbf{3 g}$ ) after 3 hr (72 96\%) (Fig. 3). On the other hand, the anti-inflammatory activity was dramatically decreased in case of isoxazole carboxylic acid amide derivatives $(\mathbf{2 2}, \mathbf{2 4}, \mathbf{2 5})$ (Fig. 4).

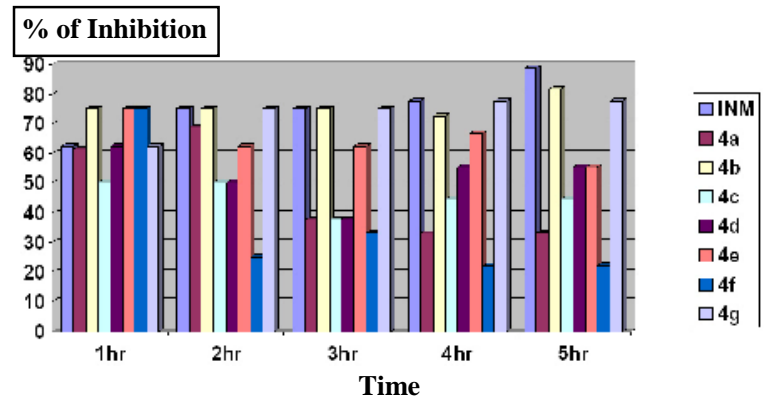

Fig. 1: Anti-inflammatory activity of 5-(p-substituted styryl)isoxazole-3-carboxylic acids 4a-g.

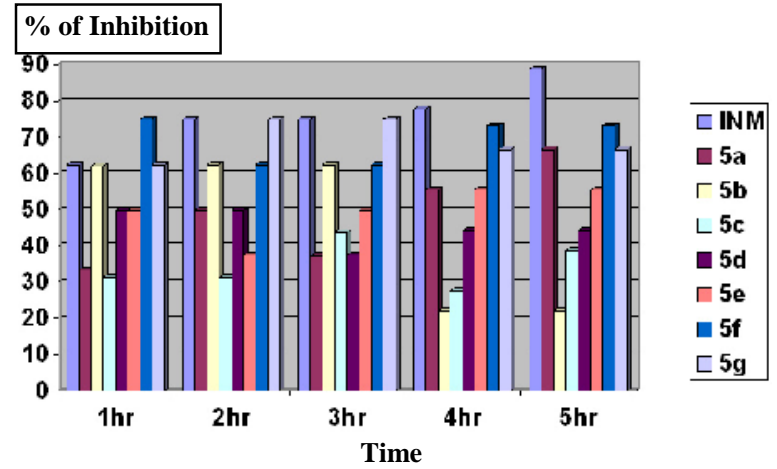

Fig. 2: Anti-inflammatory activity of 5-(p-substituted styryl)isoxazole-3-hydroxamic acids 5a-g.

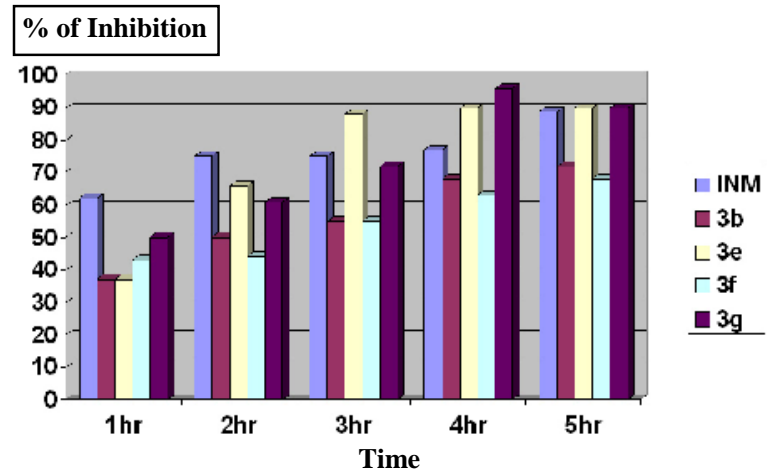

Fig. 3: Anti-inflammatory activity of 5-(p-substituted styryl)isoxazole-3-carboxylic acid ethyl esters 3b, 3e, 3f, 3g.

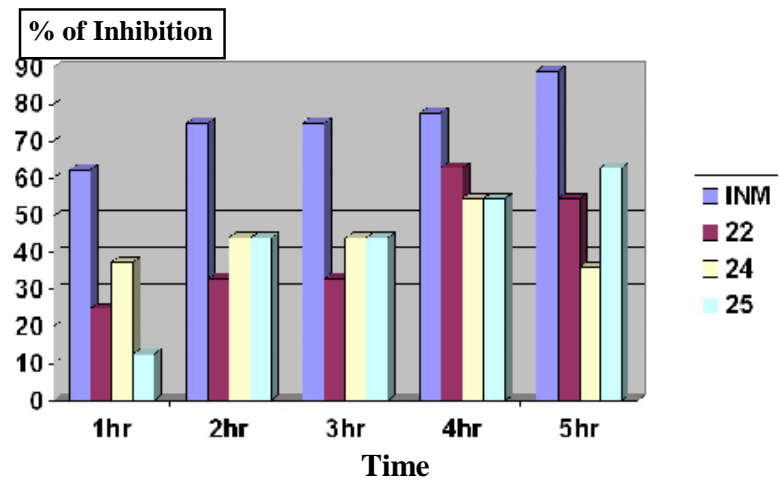

Fig. 4: Anti-inflammatory activity of 5-(p-substituted styryl)isoxazole-3-(N-substituted)carboxamides 22, 24 and 25. 
Evaluation of the anti-inflammatory activity of p-flouoro, p-nitro and p-methoxy isoxazole carboxylic acid (4b,e and f), hydroxamic acid (5b,e and $\mathbf{f})$, carboxylic acid ethyl ester (3b,e and f) and carboxylic acid amide were performed to explore the effects of derivatization of the carboxylate moiety of the target isoxazole derivatives (Fig. 5).

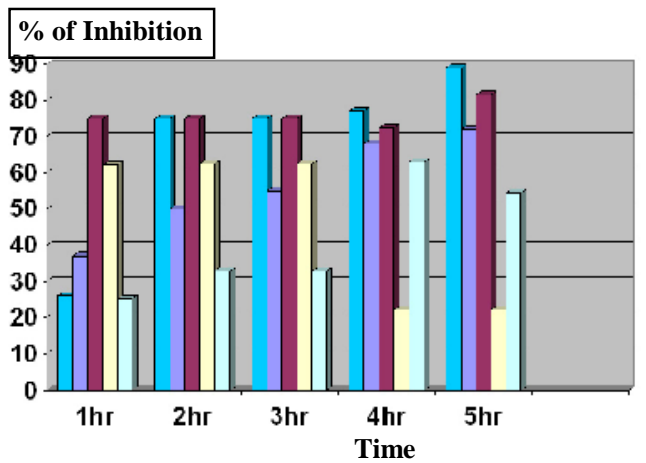

\begin{tabular}{l}
$\square$ INM \\
$\square 3 \mathrm{~b}$ \\
$\square 4 \mathrm{~b}$ \\
$\sqcup 5 \mathrm{~b}$ \\
$\sqcup 22$ \\
\hline
\end{tabular}

Fig. 5: Anti-inflammatory activity of 5-(pflourostyryl)isoxazole derivatives $\mathbf{3 b}, \mathbf{4 b}$, $\mathbf{5 b}$ and 22.

Higher inhibitory activity of inflammation was noticed to isoxazole carboxylic acids (4b and $\mathbf{e}$ ) followed by isoxazole hydroxamic acids (5b and $\mathbf{e})$, while minimum inhibitory activity of inflammation was observed to isoxazole carboxylic acid amide derivatives $(\mathbf{2 2}, \mathbf{2 4}$ and 25) at all time intervals. At 4 and $5 \mathrm{hr}$ time intervals, 5-( $p$-nitrostyryl)-isoxazole-3carboxylic acid ethyl ester (3e) exhibited higher activity than their corresponding carboxylic acid derivatives (4e, 5e and 24).

The carboxylate moiety in this series of compounds (4b,e,f) was transformed into carboxylic acid ethyl ester (3b,e and $\mathbf{f}$ ), hydroxamic acids (5b,e and $\mathbf{f})$ and carboxylic acid amides (22, 24 and 25), since a chemical derivatizations (amidation or esterification) of the carboxylate moiety in NSAIDs generates an impressive array of potent and highly selective COX-2 inhibitors. The inhibition of gastriclesions is the indicator about such activity. Generally, isoxazole carboxylic acid derivatives showed higher activity than carboxylic acid amide derivatives, which revealed the lowest activity while hydroxamic acid derivatives were noticed in between.

Examination of The stomach specimens of the treated experimental animals under scanning electron microscope afforded a highly precise, accurate and self-explanatory description of the effects of the investigated compounds on the protective as well as the mucosal layer of the gastrointestinal tract. The ulceroginicity of the compounds $\mathbf{3 g}, \mathbf{4 g}$ and $\mathbf{5 g}$ as representatives of the anti-inflammatory active compounds (ester, carboxylic acid and hydroxamic acid) and indomethacin as reference drug were examined under scan electron microscope, after 24 hours of administration of single dose $(100 \mathrm{mg} / \mathrm{kg})$ in rats. Fig. 6 illustrate scanning electromicrographs for the stomach specimens of the rats treated with a single dose of the compounds 3g (Fig. 6D), 4g (Fig. 6C), 5g (Fig. 6E), indomethacin (Fig. 6B) and control (Fig. 6A). As showed in Fig. 1B the indomethacin treated animals were characterized by complete damage of the mucous layer beside ulceration of submucosal cells. The compound $\mathbf{4 g}$ treated animals showed damage of the mucosal layer but to les extent than that with indomethacin. The animals treated with hydroxamic acid (5g) showed the least damage of the mucosal layer. This indicates that the acid derivatives have the highest ulcerogenic effect among the tested compounds, due to the direct action of acid on mucosal, the hydroxamic acid is the least one. On the other hand, isoxazole carboxylic acid ethyl ester (3g) showed a matched or higher potency relative to indomethacin with a lack of ulcerogenic effect relative to indomethacin and acid derivative $\mathbf{4 g}$.

The chemical modification of the substituents on the para position of the phenyl moiety attached to the vinyl group were designed to study the effect of variation of these substituents on the electronic and the physicochemical properties of whole molecule and subsequently the anti-inflammatory activity. From the results obtained, isoxazole carboxylic acids derivatives bearing $p$ flourophenyl group (4b) and pdimethylaminophenyl (4g) showed maximum inhibition of inflammation after one hour and persist up to $5 \mathrm{hr}$ my indicate rapid absorption and resistance to metabolism of these compounds. Since p-fluoro is an electron withdrawing substituent while pdimethylamino is an electron donating one and both maintain equipotency relative to indomethacin may reflect that the electronic properties of these substituents is not the key factor for the activity. 


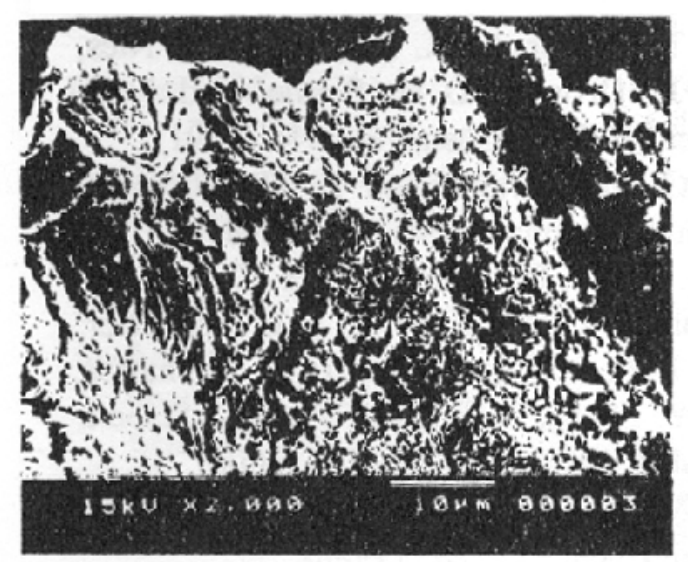

A

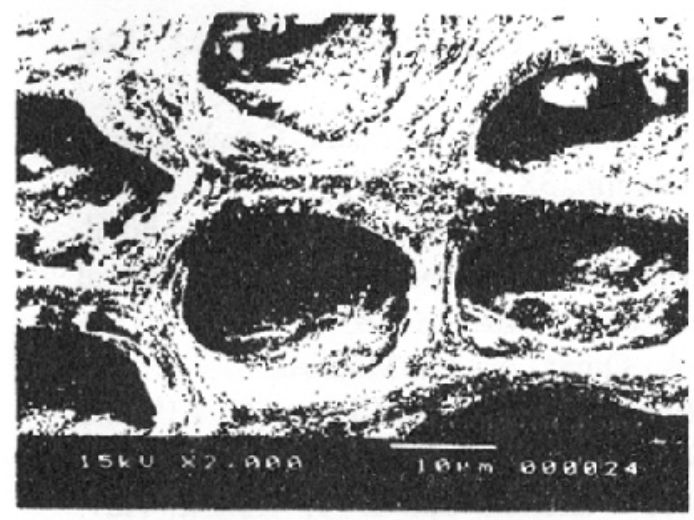

C

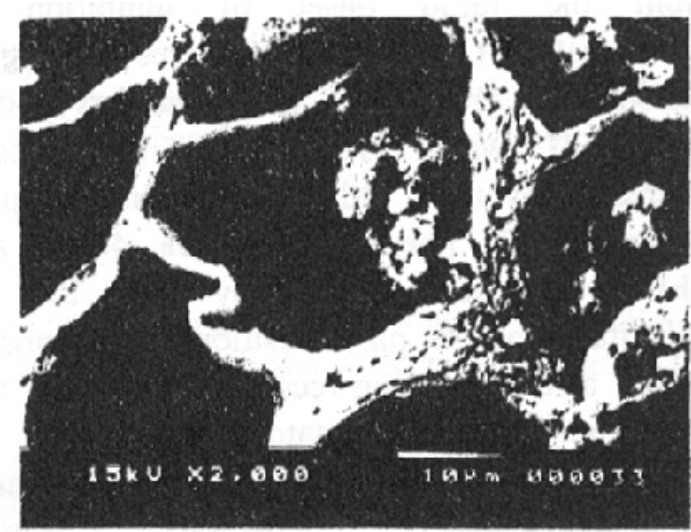

B

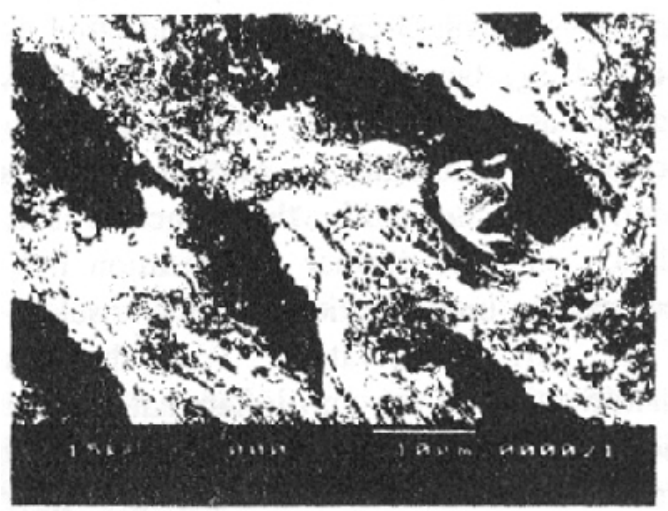

D

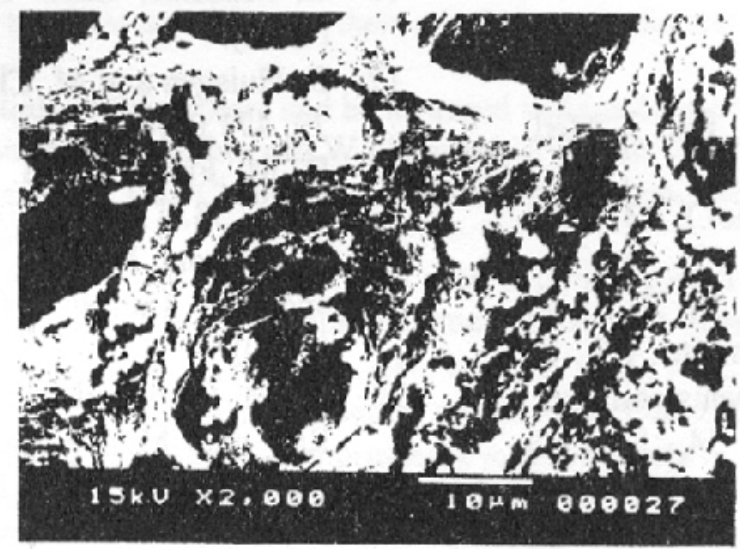

$\mathbf{E}$

Fig. 6: Scanning electromicrographs of the rats stomach specimen after route dose (100 $\mathrm{mg} / \mathrm{kg}$ ) of: A: control; B: indomethacine; C: 4g; D: 3g; E: 5g. 
The effects of the substituent on the para position of the phenyl moiety on the vulnerability to metabolism is clearly observed from the rapid onset of inhibition of inflammation (1 hr) for derivatives bearing pnitrophenyl group $\mathbf{4 e}$ and p-methoxyphenyl group $\mathbf{4 f}$ which slightly or dramatically decreased by time. Other substituents on para position of the phenyl moiety (4a, $\mathbf{4 c}, \mathbf{4 d}$ and 4h) revealed less activity my prove that the substituent at the para position of the phenyl highly affect the drug-receptor interaction and subsequently the inflammatory inhibition.

In summary, a significant antiinflammatory activity was displayed by most of the target derivatives. The anti-inflammatory activity of 5-( $p$-nitro and $p$-dimethylaminostryryl)-isoxazole-3-carboxylic acid ethyl esters (3e and $\mathbf{3 g}$ ) being favorably comparable with indomethacin in terms of potency and ulcerogenic liability. Further structural modifications to study the structure activity relation ship for further optimization may be necessary to improve the potency and selectivity of the current derivatives. With the aim of quantification of the anti-inflammatory activity and explaining the mechanism of action of these products, further investigations are now in progress.

\section{REFERENCES}

1- G. Dannhardt and W. Jiefer, Eur. J. Med. Chem., 36, 109 (2001).

2- J. R. Vane, Nature (New Biol.), 231, 232 (1971).

3- F. L. Daniel, R. B. Thomas, M. B. Amal, C. T. David, K. R. Catherine, N. E. Donald, O. F. Daniel, S. J. Denis and S. C. Jagadish, J. Med. Chem., 34, 518-525 (1991).

4- S. B. James, M. Hormos, H. H. James, R. D. James, D. D. Richard and C. W. Carter, J. Med. Chem., 30, 574-580 (1987).
5- J. M. Gibian and A. R. Galaway, Bio-Org. Chem., 1, 117 (1977).

6- D. L. Flynn, T. R. Belliotti, A. M. Boctor, D. T. Connor, C. R. Kostlan, D. E. Nies, D. F. Ortwine, D. J. Schrier and J. C. Sircar, J. Med. Chem., 34, 518 (1991).

7- J. G. Lombardino and E. H. Wiseman, Trends Pharmacol. Sci., 132 (1981).

8- J. J. Talley, D. L. Brown, J. S. Cater, M. J. Graneto, C. M. Koboldt, J. L. Masferrer, W. E. Perkins, R. S. Rogers, A. F. Shaffer, Y. Y. Zhang, B. S. Zweifel and K. Seibert, J. Med. Chem., 43, 775-777 (2000).

9- A. M. Badger and J. L. Adams, Ann. Rep. Med. Chem., 33, 203 (1998).

10- G. Soliman and L. Rateb, J. Chem. Soc., 3663, (1956).

11- C. Musante, Gazz. Chem. Ital., 72, 134, (1942).

12- C. Otukar, O. Kriz, Collect. Czech. Chem. Commum., 38, 294, (1973).

13- L. V. G. Nargund, G. R. N. Reddy and V. Hariprasad, Journal of Pharmaceutical Science, 83, 246-248 (1994).

14- F. A. Omar, N. M. Mahfouz and M. A. Rahman, Eur. J. Med. Chem., 31, 819-825 (1996).

15- B. J. Wakefield and D. J. Wright, Adv. Heterocycl. Chem., 25, 147 (1979)

16- C. Musante, Gazz. Chem. Ital., 72, 134, (1942).

17- R. Huisgen and M. Christl, Angew. Chem., 79, 471 (1967).

18- B. M. Fung and M. J. Gerace, J. Chem. Phys., 53, 1171 (1970).

19- C. F. Beam, M. C. D. Dyer, R. A. Schwarz and C. R. Hauser, J. Org. Chem., 35, 1806 (1970).

20- M. M. Campbell, N. D. P. Cosford, D. R. Rae and M. Sainsbury, J. Chem. Soc. Perkin Trans.1, 765 (1991).

21- J. Gainer, G. A. Haworth, W. Hoyle, and S. M. Roberts, Org. Magn. Reson, 8, 226 (1976). 